\title{
Gauss-Bonnet correction to Holographic thermalization: two-point functions, circular Wilson loops and entanglement entropy
}

\author{
Yong-Zhuang $\mathrm{Li}^{1}$, Shao-Feng $\mathrm{Wu}^{1,2 *}$, and Guo-Hong Yang ${ }^{1,2}$ \\ ${ }^{1}$ Department of physics, Shanghai University, \\ Shanghai, 200444, P. R. China and \\ ${ }^{2}$ The Shanghai Key Lab of Astrophysics, Shanghai, 200234, P. R. China
}

\begin{abstract}
We study the thermalization of a class of 4-dimensional strongly coupled theories dual to a 5-dimensional AdS-Vaidya spacetime with Gauss-Bonnet curvature corrections. We probe the thermalization using the two-point functions, the expectation values of circular Wilson loops and entanglement entropy. When boundary separation is small, we observe that the thermalization times of these observables have the weak dependence on the Gauss-Bonnet coupling constant $\alpha$. In addition, the growth rate of entanglement entropy density is nearly volume-independent. We also show that a new kind of swallow-tail behavior may exhibit in the thermalization of the two-point function when $\alpha$ is negative and $\ell$ is large enough. At large negative $\alpha(\alpha \lesssim-0.1)$ the relationship between the critical thermalization time of entanglement entropy and the boundary separation encounters certain "phase transition".
\end{abstract}

PACS numbers: 11.25.Tq, 12.38.Mh, 03.65.Ud

\footnotetext{
* Corresponding author. Email: sfwu@shu.edu.cn; Phone: +86-021-66136202.
} 


\section{INTRODUCTION}

The AdS/CFT correspondence [1] has been fruitful in revealing universal features of strongly coupled field theories by gravitational description. The duality has entered into the regime of far-from-equilibrium physics. In particular, the so-called "holographic thermalization" has attracted many interest, which is well motivated by the demand of describing the fast thermalization of the quark gluon plasma produced in heavy ion collisions at the Relativistic Heavy Ion Collider [2, 3] and of describing some condensed matter systems prepared under quantum quenches [4].

In this note, we will investigate the thermalization process of a class of strongly coupled conformal field theories (CFTs) by its dual gravity with Gauss-Bonnet (GB) curvature corrections. Generally, the higher derivative corrections appear in any quantum gravity theory (like string theory) from quantum or stringy effect. These corrections may be holographic dual to $1 / N$ or $1 / \lambda$ corrections in some gauge theories. In terms of the standard correspondence between type IIB string theory on $A d S_{5} \times S^{5}$ and the $\mathcal{N}=4$ super Yang-Mills theory (SYM) theory, the stringy effect gives the leading order $1 / \lambda$ corrections, which has the form of $\alpha^{\prime 3} R^{4}$, rather than $R^{2}$. However, the quadratic curvature corrections may also arise in the framework of string theory. Consider the dual between the $\mathcal{N}=2 S p(N)$ gauge theory (with 4 fundamental and 1 antisymmetric traceless hypermultiplets) and type IIB string theory on $A d S_{5} \times X^{5}$ with $X^{5} \simeq S^{5} / \mathbb{Z}_{2}[5]$. The curvature squared term arises in the effective action on the D7 and O7 world-volumes [6] and leads to certain subleading $1 / N$ corrections. Note that the corrections allow independent values of the two central charges $a$ and $c$ of the dual field theory, in contrast to the $\mathcal{N}=4$ SYM where $a=c$. This feature is essential for the violation of the well-known Kovtun-Starinets-Son (KSS) bound of the ratio of the shear viscosity $\eta$ to the entropy density $s\left[\begin{array}{ll}7 & 9\end{array}\right]$. Beside its relation with string theory mentioned above, there are also some motivations to involve the GB curvature corrections [45] in holographic theories, two of which are particular for the holographic thermalization. i) Keeping in mind the vastness of the string landscape, one could not rule out certain situations in which GB curvature is dominated in all the higher derivative corrections [7]. ii) Comparing with the other higher curvature theory of gravity, the equations of motion of GB gravity are only second order in derivatives. This makes the holographic calculations being easily dealt with. iii) It was found that GB gravity captures the causality constraints [7, 8] which can 
be also inferred from the positivity of the energy in CFT analysis [10]. Such fundamental correspondence conveys a piece of strong evidence supporting the AdS/CFT conjecture. iv) Since the KSS bound is not effective in theories dual to GB gravity, it promotes to treat GB correction as a dangerous source of violation for the features which is universal in usual holographic models [11]. For instance, the universality of the ratio of gap frequency over critical temperature on holographic superconductors breaks in the presence of the GB coupling [12]. v) The exact black hole solution of GB gravity, particularly the Vaidya type solution, have been constructed [13]. This paves the way for our study on holographic thermalization. vi) Entanglement entropy (EE) is an important non-local observable of holographic thermalization. However, there is not yet a general holographic prescription for computing EE in higher curvature theory of gravity (such as the one with $R^{4}$ correction from type IIB string theory), except in the case of GB gravity [14 16].

We will study three non-local observables, including the two-point correlation function, the (circular) Wilson loop expectation values and the EE (with circular entangling surfaces). They can be evaluated in the saddle point approximation in terms of some extremal geometric objects in the bulk. We will adapt the simple AdS-Vaidya model [17-27], which describes a homogeneous [46] falling thin shell of null dust and is a good quantitative approximation of the background generated by the perturbation of a time-dependent scalar field [29] and of the model of Ref. [30]. In term of Vaidya models, some interesting features of holographic thermalization have been found. For instance, i) the thermalization process is top-down, i.e. the high momentum modes thermalize faster than long wavelength ones. This is contrary to the thermalization behavior of the weak field theory [31] but is very natural in the Vaidya models, where the collapsing shell from the boundary passes the extremal surface of the smaller probe more faster than the one of the larger probe. ii) EE thermalizes slowest among the probes and sets the time-scale for thermalization. This feature may also be understood intuitively since other observables are sensitive only to a subset of all degrees of freedom of the field theory which are involved in the calculation of entropy [3]. iii) It was observed that the evolution of holographic EE includes an intermediate stage during which it is a simple linear function of time. The linear regime is not obvious when the boundary separation is small, but it will be when the boundary separation increases. This result matches well with the behavior seen in $d=2$ CFTs [32, 33]. Also it is consistent with the evolution of coarse-grained entropy in nonlinear dynamical systems. In particular, in 
classical 4-dim SU(2) lattice gauge theory, the linear growth rate of coarse-grained entropy, that is generally described by the Kolmogorov-Sinaï entropy rate [34], is shown to be an extensive quantity [35]. For strongly coupled field theory with gravity dual, it has been found [20] that the growth rate of EE density in $\mathrm{d}=2 \mathrm{CFT}$ is also nearly volume-independent for small boundary volumes. For large volumes, however, the growth rate of EE approaches a constant limit[47]. Recently, the large volume behavior of EE has been studied in very general situations [11, 24, $\underline{36}]$.

We will focus on studying how the thermalization time of various probes and the growth of EE at small volume are affected by the GB curvature corrections. It is interesting that we find out both the universal features which are independent with the curvature corrections and the new thermalization behavior that is induced by the curvature corrections.

It should be noted that the higher curvature corrections to holographic thermalization have been also discussed using the quasi-static approximation [37, 38] and the Vaidya model [11, 24, 26, 27], respectively. In particular, Ref. [26] investigated the thermalization time scale in SYM plasmas with the leading type IIB string theory corrections. It was found that both $\alpha^{\prime}$ and $1 / N$ contributions decrease just a very little the thermalization time of UV modes. We will also show the weak effects of GB correction on the thermalization time when the boundary separation is small. Moreover, the GB correction to the two-point function and the rectangular Wilson loop has been studied in Ref. [27]. We will compare our results with theirs at the last section.

\section{THE GB GRAVITY}

Consider the 5D GB gravity with a negative cosmological constant $\Lambda$ [39],

$$
I=\frac{1}{16 \pi G_{N}^{(5)}} \int d^{5} x \sqrt{-g}\left[-2 \Lambda+\mathcal{R}+\frac{\alpha}{2} L^{2} \mathcal{L}_{G B}\right],
$$

where

$$
\Lambda=-\frac{6}{L^{2}}, \quad \mathcal{L}_{G B}=\mathcal{R}^{2}-4 \mathcal{R}_{\mu \nu} \mathcal{R}^{\mu \nu}+\mathcal{R}_{\mu \nu \rho \sigma} \mathcal{R}^{\mu \nu \rho \sigma},
$$

$L$ is AdS spacetime radius and $\alpha$ is GB coupling constant. It should be noted that there exists

a constraint $-\frac{7}{36} \leq \alpha \leq \frac{9}{100}$ by respecting the causality of dual field theory on the boundary [7, 8] or preserving the positivity of the energy flux in CFT analysis [10]. However, we still 
calculate three probes with $-\frac{7}{36} \leq \alpha \leq \frac{1}{4}$. As a result, one could extract some information on how the violation of the causality constraint of $\alpha$ influences the thermalization.

For simplicity we will set $L=1$ hereafter. The action gives the equation of gravitational field

$$
\mathcal{R}_{\mu \nu}-\frac{1}{2} g_{\mu \nu} \mathcal{R}=-\Lambda g_{\mu \nu}+\frac{\alpha}{2} \mathcal{H}_{\mu \nu}
$$

where

$$
\mathcal{H}_{\mu \nu}=\frac{1}{2} g_{\mu \nu} \mathcal{L}_{G B}-2 \mathcal{R} \mathcal{R}_{\mu \nu}+4 \mathcal{R}_{\mu \gamma} \mathcal{R}^{\gamma}{ }_{\nu}+4 \mathcal{R}_{\gamma \delta} \mathcal{R}_{\mu \nu}^{\gamma{ }^{\delta}{ }_{\nu}}-2 \mathcal{R}_{\mu \gamma \delta \lambda} \mathcal{R}_{\nu}{ }^{\gamma \delta \lambda}
$$

Eq. (3) has a static black brane solution [39]

$$
d s^{2}=-r^{2} f(r) d t^{2}+\frac{1}{r^{2} f(r)} d r^{2}+\frac{r^{2}}{L_{A d S}^{2}} d \mathbf{x}^{2},
$$

where $f(r)=\frac{1}{2 \alpha}\left[1-\sqrt{1-4 \alpha\left(1-\frac{r_{h}^{4}}{r^{4}}\right)}\right], r_{h}$ is event horizon radius, and the effective AdS radius is given by $L_{A d S}^{2}=\frac{1+\sqrt{1-4 \alpha}}{2} . \mathbf{x}=\left(x_{1}, \ldots, x_{d-1}\right)$ correspond to the spatial coordinates on the boundary.

To model the thermalization processes of strongly coupled field theories by a homogeneous falling thin shell of null dust in AdS spacetime, we invoke a Vaidya type solution [13]:

$$
d s^{2}=\frac{1}{z^{2}}\left[-f(z, v) d v^{2}-2 d z d v+\frac{1}{L_{A d S}^{2}} d \mathbf{x}^{2}\right]
$$

where $f(z, v)=\frac{1}{2 \alpha}\left[1-\sqrt{1-4 \alpha\left(1-m(v) z^{4}\right)}\right]$ and $z$ is the inverse of radial coordinate $r$. The mass function is

$$
m(v)=\frac{M}{2}\left[1+\tanh \left(\frac{v}{v_{0}}\right)\right],
$$

where $M$ denotes the mass of the black brane for $v>v_{0}$ and $v_{0}$ represents a finite shell thickness. We will be interested in the zero thickness limit, which means to set the energy deposition on the boundary as instantaneous. But for later numerical convention, we set $v_{0}=0.01$. Moreover, we will set $M=1$ and the meaning will be interpreted below.

One may notice that there are various forms of the GB black brane metric in references [48], which are related by a simple coordinate transformation, namely the effective AdS radius may disappear or appear in certain component of metric. The difference does not change the dimensionless quantities, such as $\eta / s$ in natural units. However, one should be careful to deal with the dimensional quantities like the temperature, boundary separation and thermalization time. We select the form of Eq. (5) because of two reasons. One is that 
it is asymptotically AdS which respects the same symmetry of the CFTs. The other is that

the Hawking temperature $T=\frac{r_{h}}{\pi}=\frac{M^{1 / 4}}{\pi}$ is independent with $\alpha$ if we fix $M=1$. Consider that we use the collapse of a massless shell to model a certain thermal quench, or a sudden injection of energy due to a heavy-ion collision, and this energy will be involved in the mass $M$ ultimately [26]. Thus, if we adapt the metric Eq. (5) and fix $M=1$, we have fixed the injected energy and the ultimate equilibrium temperature simultaneously, which makes the comparison of thermalization processes with different $\alpha$ more reasonably. Note that these two good situations do not happen if one does not select the form of the metric suitably. Also see Ref. [26], in which $M$ can be fixed but $T$ will be dependent with the 't Hooft coupling, vice versa.

\section{THE NON-LOCAL OBSERVABLES}

In order to explore the dynamics and the scale dependence of thermalization processes, the local observables can not provide sufficient information. Here we will study the equal-time two-point function, Wilson loop expectation values and EE. Using the AdS/CFT correspondence, the former two can be calculated by the space-like geodesic and the extremal surface which extend in the bulk and end on the boundary, respectively. The EE in the field theory dual to GB gravity can also be obtained by extremizing some geometric quantities in the bulk but is a little more complicated, since the holographic formula of EE in GB gravity has the nontrivial correction (not same as the Wald entropy) to the one of Einstein gravity [14 16, 41].

\section{A. The two-point function}

Consider the space-like geodesic connecting two boundary points $\left(t, x_{1}\right)=\left(t_{0},-\ell / 2\right)$ and $\left(t, x_{1}\right)=\left(t_{0}, \ell / 2\right)$. We have set that all other spatial directions are identical at the two end points. One can parameterize such a geodesic by $v=v(x), z=z(x)$ where we have denoted $x_{1}=x$. The length of geodesic is given by extremizing the action

$$
\mathcal{L}\left(\ell, t_{0}\right)=\int_{-\ell / 2}^{\ell / 2} d x \frac{1}{z} \sqrt{\frac{1}{L_{A d S}^{2}}-2 z^{\prime} v^{\prime}-f(z, v) v^{\prime 2}},
$$


where the prime indicates derivative with respect to $x$. To obtain the minimal length of the geodesic, we need to solve the two equations of motion that are derived from Eq. (8):

$$
\begin{gathered}
2 L_{A d S}^{2} z z^{\prime \prime 2}+\left\{L_{A d S}^{2} z v^{\prime}\left(v^{\prime} \frac{\partial f}{\partial v}+2 z^{\prime} \frac{\partial f}{\partial z}\right)-2 f^{2} L_{A d S}^{2} v^{\prime 2}+f\left[2+L_{A d S}^{2} v^{\prime}\left(z v^{\prime} \frac{\partial f}{\partial z}-4 z^{\prime}\right)\right]\right\}=0, \\
2 z v^{\prime \prime}-\left\{\frac{2}{L_{A d S}^{2}}+v^{\prime}\left[v^{\prime}\left(z \frac{\partial f}{\partial z}-2 f\right)-4 z^{\prime}\right]\right\}=0 .
\end{gathered}
$$

Keeping in mind with the symmetry of the geodesic, one can impose the boundary conditions

$$
z(0)=z_{*}, v^{\prime}(0)=v_{*}, z^{\prime}(0)=0, v^{\prime}(0)=0 .
$$

The two parameters $z_{*}$ and $v_{*}$ are determined by the constrain equations

$$
z\left( \pm \frac{\ell}{2}\right)=z_{0}, v\left( \pm \frac{\ell}{2}\right)=t_{0},
$$

where $z_{0}$ is an IR bulk cut-off.

Using Eq. (11) and Eq. (12), we can numerically solve Eqs. (99) and (10) to calculate the minimal length of the geodesic by Eq. (8). We plot $\delta \overline{\mathcal{L}}\left(\ell, t_{0}\right)=\frac{\delta \mathcal{L}\left(\ell, t_{0}\right)-\delta \mathcal{L}_{\text {thermal }}(\ell)}{\ell}$ as a function of the boundary time $t_{0}$, the boundary separation $\ell$ and GB coupling $\alpha$ in Fig. 1, The regularized length $\delta \mathcal{L}\left(\ell, t_{0}\right)$ is given by subtracting the cut-off dependent part $\mathcal{L}_{\text {div }}=$ $-2 L_{A d S} \log z_{0}$ from Eq. (8). Note that $\mathcal{L}_{\text {div }}$ can be obtained in the vacuum background. In addition, the subscript "thermal" denotes the regularized thermal equilibrium value that can be calculated in the background of pure black branes.

One key quantity to characterize the thermalization process is the thermalization time. In general, the real thermalization time $\tau_{\text {real }}$ should be defined as the time when the observable reaches the thermal value. There are also other definitions of thermalization time which can describe certain aspects of the thermalization process [20]. i) The half-thermalization time, at which the observable reaches half of their equilibrium value; ii) the rapid thermalization time, at which thermalization proceeds most rapidly; iii) the critical thermalization time $\tau_{c r i t}$, at which the tip of the extremal line or surface grazes the middle of the shell at $v=0$. Beside the real thermalization time that can be read from Fig. 1, we also have interested in the critical time with the mind of its two features. First, $\tau_{\text {crit }}$ can be determined by the black brane geometry outside the infalling shell:

$$
\tau_{\text {crit }}=\int_{z_{0}}^{z_{*}} \frac{d z}{f(z)},
$$



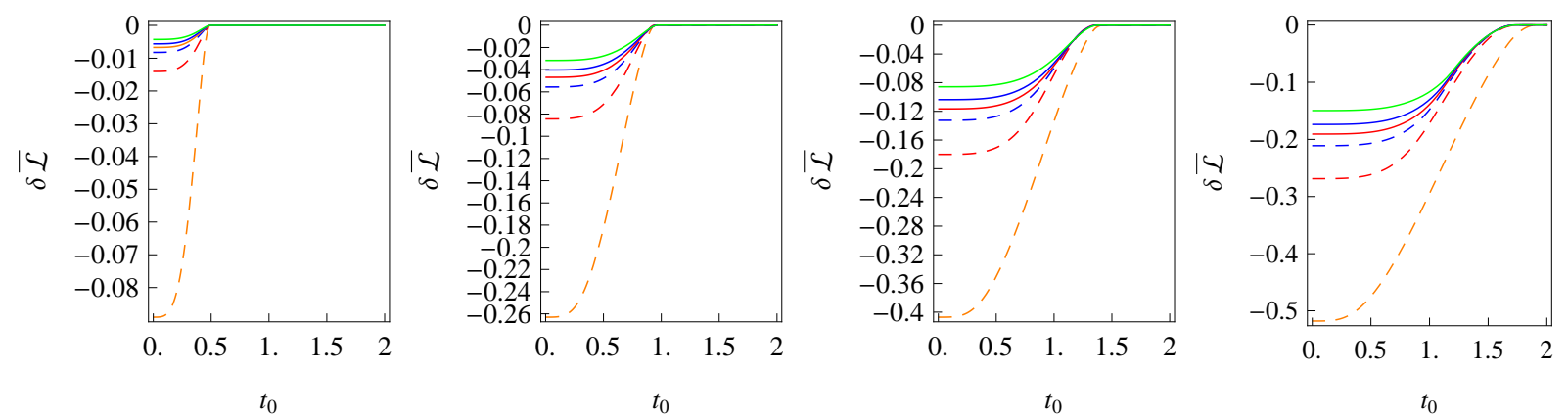

FIG. 1: $\delta \overline{\mathcal{L}}$ as a function of $t_{0}$ for boundary scale $\ell=1,2,3,4$ (from left to right) with different GB coupling constants $\alpha=-0.19,-0.1,-0.05,0,0.1,0.24$ according to the green, blue, red, blue-dashed, red-dashed, orange-dashed lines, respectively. For simplicity we will adapt the same identification among curves and GB couplings from this figure to Fig. 6.

where $z_{*}$ depends on the boundary separation and $f(z)=\frac{1}{2 \alpha}\left[1-\sqrt{1-4 \alpha\left(1-M z^{4}\right)}\right]$. One can find that the more complicated dynamical Vaidya geometry is not necessary to compute $\tau_{\text {crit }}$. Consequently, the behavior of $\tau_{\text {crit }}$ can be analytically analyzed in certain extent, as we will show later. Second, it has been pointed out in [21] that $\tau_{\text {crit }}$ provides a lower bound of $\tau_{\text {real }}$ in general[49]. Moreover, we have checked numerically that $\tau_{\text {crit }} \simeq \tau_{\text {real }}$ for three probes when the boundary separation is small enough. Thus one may use $\tau_{\text {crit }}$ to suspect or understand some properties of $\tau_{\text {real }}$.

From Fig. 1, one can find a nontrivial result that $\tau_{\text {real }}$ is nearly independent with the GB curvature effect when $\ell$ is small, particularly for the cases with $\alpha \neq 0.24$ (which exceeds far more than the causality bound $\left.-\frac{7}{36} \leq \alpha \leq \frac{9}{100}\right)$. This behavior also appears in the critical thermalization time. To be more clear, we plot the relative critical thermalization time $\delta \bar{\tau}_{\text {crit }}\left(\equiv \frac{\tau_{c r i t}-(\ell / 2)}{\ell / 2}\right)$ as a function of boundary separation $\ell$ in the left panel of Fig. 2, Note that the right panel about the maximal growth rate is plotted for later comparison.

Beside the small dependence of critical thermalization time on the $\alpha$, we also find two interesting results. i) The critical thermalization time has the non-monotonic dependence on the GB coupling constant $\alpha$. When the boundary separation is small, the critical thermalization time decreases as $\alpha$ increases. But it seems that there is the opposite behavior when the boundary separation is large enough. One can see the tendency more clearly for the case of Wilson loop in next subsection. It should be noted that our numerical precision of $\tau_{\text {real }}$ is not high enough when $\ell$ is large, so we can not identify the non-monotonic feature 

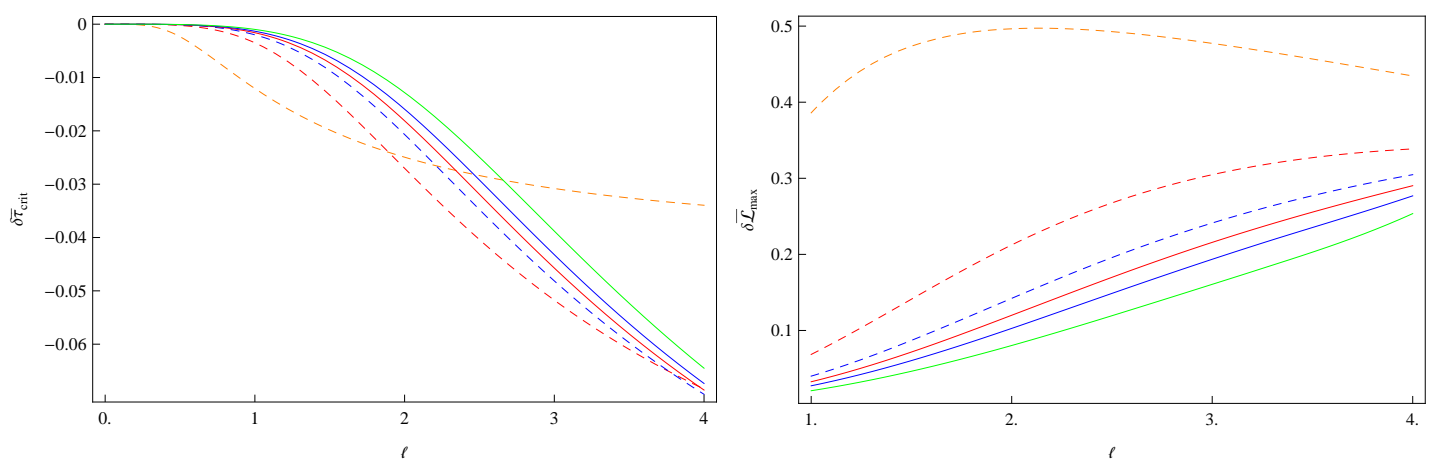

FIG. 2: (Left) The relative critical thermalization time $\delta \bar{\tau}_{\text {crit }}\left(\equiv \frac{\tau_{c r i t}-(\ell / 2)}{\ell / 2}\right)$ as a function of spatial scale $\ell$ with different $\alpha$. (Right) Maximal growth rate of two-point function $\left(\delta \overline{\mathcal{L}}_{\max }\right)$ as a function of boundary scalae $\ell$.

from $\tau_{\text {real }}$. But the behavior of $\delta \bar{\tau}_{\text {crit }}$ gives a hint that $\tau_{\text {real }}$ could have the non-monotonic feature. ii) For the small boundary separation the relative critical thermalization time is nearly vanishing. In other word, the critical thermalization time saturates the causality bound $\tau_{\text {crit }} \sim \frac{\ell}{2}$ [17]. We note that this feature can be understood easily, since the geodesic with small boundary separation in the static black brane will only extend near the boundary. There, the metric is asymptotically AdS and can be written as

$$
d s^{2}=\frac{1}{L_{A d S}^{2} z^{2}}\left[-d v^{2}-2 L_{A d S}^{2} d v d z+d \mathbf{x}^{2}\right]
$$

For the two-point function one can calculate $z_{*}$ using the static metric (14) and solving the equation of motion analytically. Thus, we have

$$
z(x)=\frac{\sqrt{L_{A d S}^{4} z_{*}^{2}-x^{2}}}{L_{A d S}^{2}}, z_{*}=\frac{R_{*}}{L_{A d S}^{2}}
$$

where $R_{*}=\ell / 2$. Consequently, the critical thermalization time can be given by

$$
\tau_{\text {crit }}=\int_{z_{0}}^{z_{*}} L_{A d S}^{2} d z \propto L_{A d S}^{2} z_{*}=R_{*}=\ell / 2 .
$$

\section{B. The Wilson loop}

For the circular Wilson loop one can choose a 2-dimensional plane $\left\{x_{1}, x_{2}\right\}$ on the boundary in which this loop is described using the polar coordinate $\{\rho, \phi\}$. Then the minimal area surface can be represented by $z(\rho)$ and $v(\rho)$ with respect to the azimuthal symmetry in the 
$\phi$-direction. The area function is given by

$$
\mathcal{A}\left(R, t_{0}\right)=\int_{0}^{R} d \rho \frac{\rho}{L_{A d S} z(\rho)^{2}} Q(\rho)
$$

where $Q(\rho)=\sqrt{\frac{1}{L_{A d S}^{2}}-2 z^{\prime} v^{\prime}-f(z, v) v^{\prime 2}}$ and ${ }^{\prime} \equiv \frac{d}{d \rho}$. The equations of motion are given by

$$
\begin{aligned}
2 L_{A d S}^{2} \rho z z^{\prime \prime} & -4 L_{A d S}^{2} \rho f^{2} v^{\prime 2}-L_{A d S}^{2} z\left[4 L_{A d S}^{2} v^{\prime} z^{\prime 2}-\rho v^{\prime 2} \frac{\partial f}{\partial v}-2 z^{\prime}\left(1+\rho v^{\prime} \frac{\partial f}{\partial z}\right)\right] \\
& -f L_{A d S}^{2} v^{\prime}\left[2 z^{\prime}\left(4 \rho+L_{A d S}^{2} z v^{\prime}\right)-\rho z v^{\prime} \frac{\partial f}{\partial z}\right]+4 f \rho=0 \\
L_{A d S}^{2} \rho z v^{\prime \prime} & -\frac{1}{2} L_{A d S}^{2} \rho z v^{\prime 2} \frac{\partial f}{\partial z}+\left(2 \rho-L_{A d S}^{2} z v^{\prime}\right)\left[L_{A d S}^{2} v^{\prime}\left(f v^{\prime}+2 z^{\prime}\right)-1\right]=0 .
\end{aligned}
$$

To avoid a numerical issue at $\rho=0$, we solve Eq. (18) and Eq. (19) in the neighborhood of the midpoint by expanding around $\rho=0$ to quadratic order

$$
z(\rho)=z_{*}-\frac{f\left(z_{*}, v_{*}\right)}{2 L_{A d S}^{2} z_{*}} \rho^{2}, v(\rho)=v_{*}+\frac{\rho^{2}}{2 z_{*} L_{A d S}^{2}} .
$$

Eq. (20) will be used as the boundary conditions. Thus, we can plot $\delta \overline{\mathcal{A}}=\frac{\delta \mathcal{A}\left(R, t_{0}\right)-\delta \mathcal{A}_{\text {thermal }}(R)}{\pi R^{2}}$ as a function of the boundary time $t_{0}$ for different circular Wilson loop radius $R$ and GB coupling constant $\alpha$ in Fig. 3. Note that we have regulated the minimal area by subtracting the cut-off dependent piece:

$$
\delta \mathcal{A}\left(R, t_{0}\right)=\mathcal{A}\left(R, t_{0}\right)-\mathcal{A}_{\text {div }}(R)
$$

where $\mathcal{A}_{\text {div }}(R)=\frac{R}{z_{0}}$.

Repeating the analysis performed for two-point functions, we plot the relative critical thermalization time $\delta \bar{\tau}_{c r i t}\left(\equiv \frac{\tau_{c r i t}-R}{R}\right)$ and the maximal growth rate of minimum area density $\delta \overline{\mathcal{A}}_{\text {max }}$ in Fig. 4. The pictures show the similar behavior with the two-point function. Note that one can check Eq. (15) and (16) holding for the case of Wilson loops. In this case $R_{*}=R$.

\section{Holographic EE}

It has been proposed that the EE of a spatial region $V$ in a $d$-dimensional CFT with gravity dual is given by [42]

$$
S(V)=\frac{1}{4 G_{N}^{d+1}} \int_{\Sigma} \sqrt{h}
$$



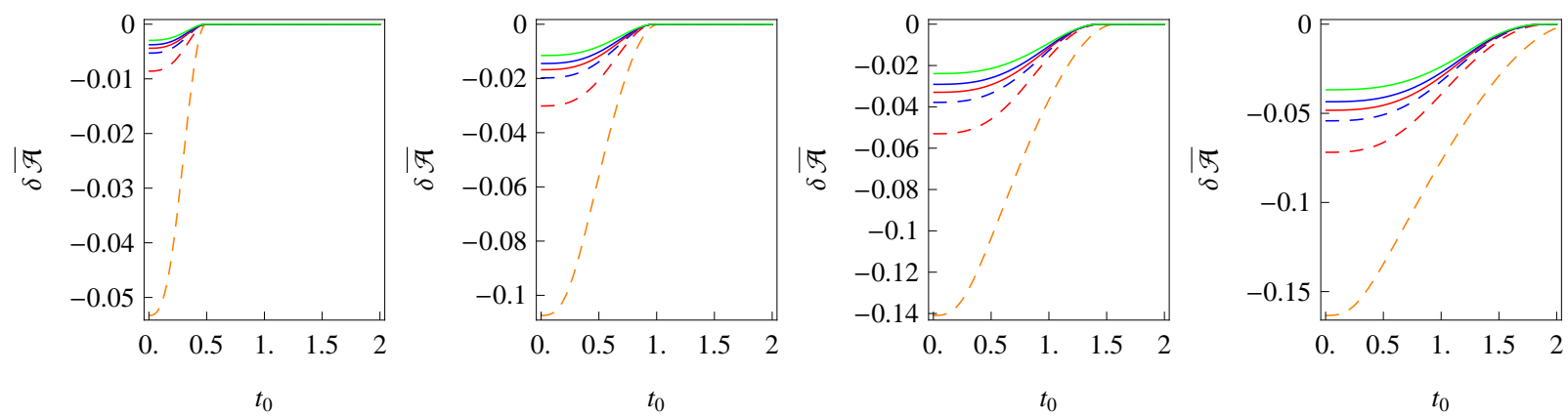

FIG. 3: $\delta \overline{\mathcal{A}}$ as a function of $t_{0}$ for circular Wilson loop radii $R=0.5,1,1.5,2$ (from left to right) with different $\alpha$.
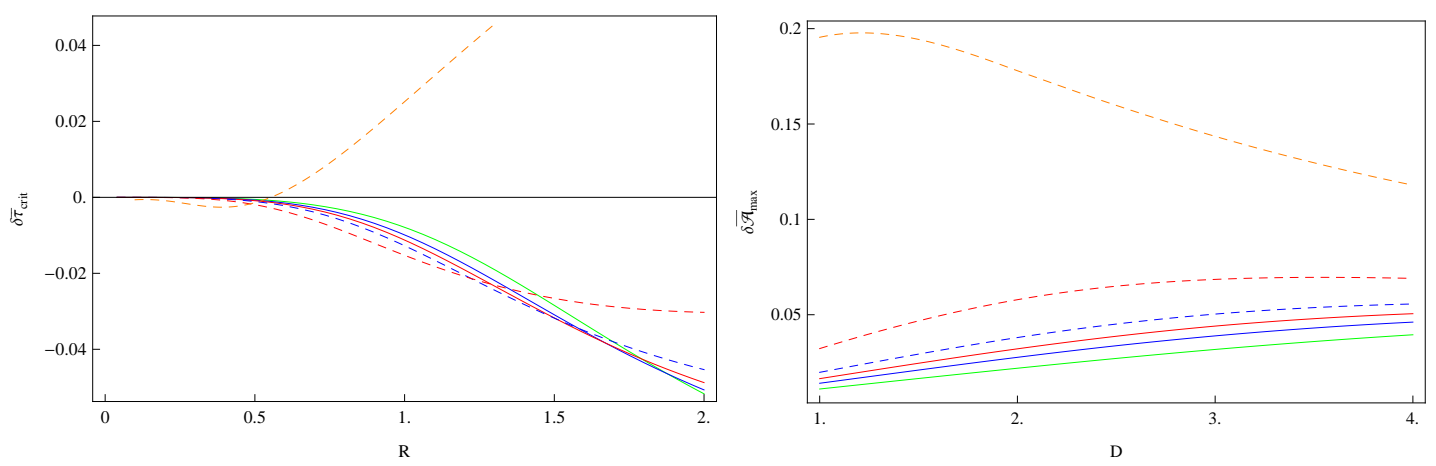

FIG. 4: (Left) The relative critical thermalization time $\delta \bar{\tau}_{c r i t}$ as a function of spatial scale $R$ with different $\alpha$. (Right) Maximal growth rate of Wilson loop density $\left(\delta \overline{\mathcal{A}}_{\max }\right)$ as a function of diameter of entangled region.

where $\Sigma$ is the minimal surface whose boundary coincides with the boundary of the region $V$ and $h$ corresponds to the determinant of the induced metric of the minimal surface. In 5D GB gravity, Eq. (22) should be generalized to be the form of [14, 15]

$$
S(V)=\frac{1}{4 G_{N}^{5}} \int_{\Sigma} d x^{3} \sqrt{h}\left(1+\alpha L^{2} \mathcal{R}_{\Sigma}\right)+\frac{\alpha L^{2}}{2 G_{N}^{5}} \int_{\partial \Sigma} d x^{2} \sqrt{\sigma} \mathcal{K}
$$

where $\mathcal{R}_{\Sigma}$ is the induced scalar curvature of $\Sigma . \sigma$ is the determinant of the induced metric on the boundary of $\Sigma$. $\mathcal{K}$ is the trace of its extrinsic curvature. In fact, the last term is the Gibbons-Hawking term which provide a good variational principle in extremizing this functional.

Defining a unit normal vector of $\partial \Sigma$ in $\Sigma$ by $n^{a}$, one can evaluate the extrinsic curvature

$$
\mathcal{K}=\left.\sigma^{a b} \nabla_{a} n_{b}\right|_{z=z_{0}}
$$


The induced metric on $\Sigma$ is given by

$$
d s_{\Sigma}^{2}=\frac{Q^{2}(\rho)}{z^{2}} d \rho^{2}+\frac{1}{z^{2} L_{A d S}^{2}} d \Omega^{2}
$$

Then the normal unit vector is given by $n_{a}=\sqrt{h_{\rho \rho}} \delta_{\rho a}$. And the volume function is given by

$$
\mathcal{V}\left(R, t_{0}\right)=4 \pi \int_{0}^{R}\left[\frac{\rho^{2}}{z^{3} L_{A d S}^{2}} Q(\rho)+q(\rho)\right] d \rho^{2}
$$

where

$$
q(\rho)=\frac{2 \alpha}{z^{3} L_{A d S}^{2} Q(\rho)}\left\{z^{2}\left[2-f(z, v) L_{A d S}^{2} v^{\prime 2}\right]-2 z\left(\rho+z L_{A d S}^{2} v^{\prime}\right) z^{\prime}+\rho^{2} z^{\prime 2}\right\} .
$$

Again, we will use the method in Section III.B by expanding the equations of motion (They can be given by Eq. (25) but we will not give out the cumbersome result clearly.) at a point $\rho_{p}$ near the midpoint to quadratic order. Then one gets the boundary conditions

$$
z(\rho)=z_{*}-\frac{f\left(z_{*}, v_{*}\right)}{2 L_{A d S}^{2} z_{*}} \rho^{2}, v(\rho)=v_{*}+\frac{\rho^{2}}{2 z_{*} L_{A d S}^{2}} .
$$

For our aim, we subtract the volume functional Eq. (25) with the cut-off dependent piece

$$
\delta \mathcal{V}\left(R, t_{0}\right)=\mathcal{V}\left(R, t_{0}\right)-\mathcal{V}_{\text {div }}(R)
$$

where the divergent term is given by

$$
\mathcal{V}_{\text {div }}(R)=2 \pi\left[\frac{R^{2}}{z_{0}^{2}} a+b \log \left(\frac{z_{0} L_{A d S}^{2}}{2 R}\right)\right],
$$

with $a=\frac{3-2 L_{A d S}^{2}}{L_{A d S}}, b=6 L_{A d S}^{5}-5 L_{A d S}^{3}$.

In Fig. 5 we plot $\delta \overline{\mathcal{V}}=\frac{\delta \mathcal{V}\left(R, t_{0}\right)-\delta \mathcal{V}_{\text {thermal }}(R)}{4 \pi R^{3} / 3}$ as a function of boundary time $t_{0}$ for various $R$ and $\alpha$. The relative critical thermalization time $\delta \bar{\tau}_{c r i t}$ is also plotted in the left panel of Fig. 6. For small $R$ the thermalization time $\tau_{\text {crit }}$ is linear with $R$. This can be understood since Eqs. (15) and (16) also hold for EE with $R_{*}=R$. Meanwhile, one may notice that the relationship between $\delta \bar{\tau}_{c r i t}$ and $R$ encounters certain "phase transition" at certain large negative $\alpha$, provided that we take $R$ as an order parameter[50] and $\delta \bar{\tau}_{\text {crit }}$ as certain "free energy". To be more clear, see Fig. 7 and one can read the critical coupling constant as $\alpha \lesssim-0.1$. In particular, note that the $\tau_{\text {crit }}$ of EE violates the causal bound (i.e. $\tau_{\text {crit }} \geqslant R$ ) for the large negative $\alpha$ when $R$ is in a certain interval. Also note that the causal bound is violated for other two probes in Einstein gravity, while not for EE [20].

Now it is time to compare the right panels of Figs. 2, 4, and 6. One can find that for small boundary region the growth rate of EE density is nearly volume-independent, but that is not the case for other two probes. 

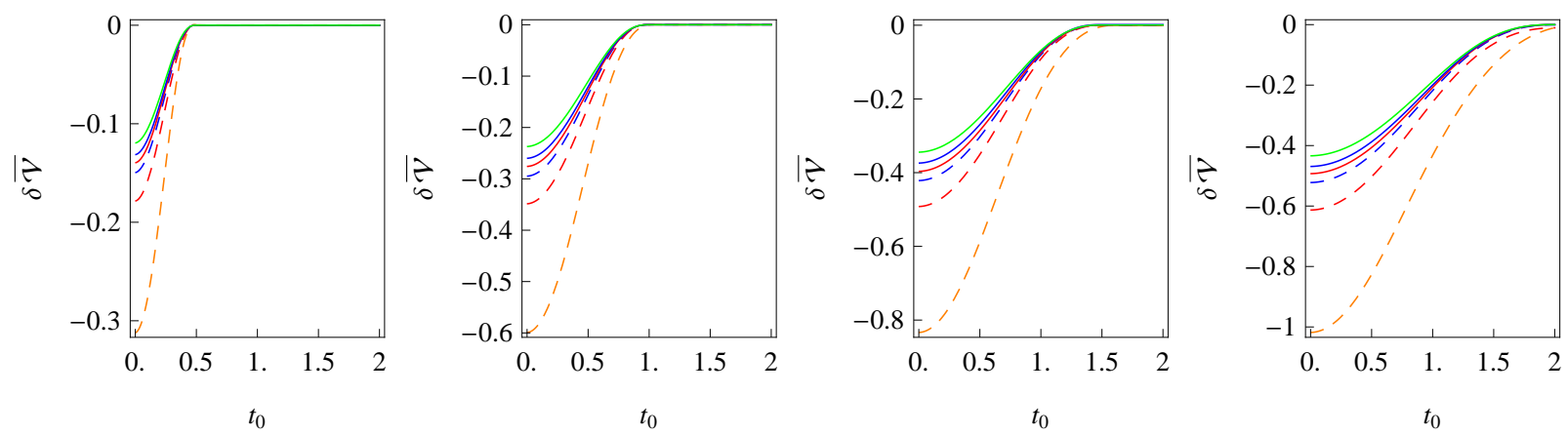

FIG. 5: $\delta \overline{\mathcal{V}}$ as a function of boundary time $t_{0}$ for boundary radii $R=0.5,1,1.5,2$ (from left to right) with different $\alpha$.
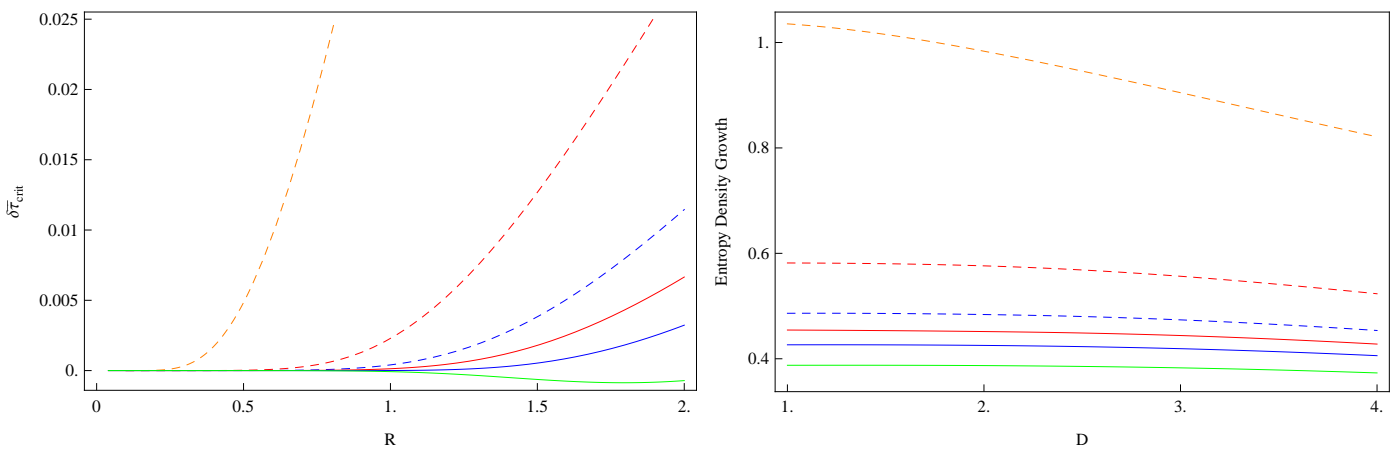

FIG. 6: (Left) The relative critical thermalization time $\delta \bar{\tau}_{\text {crit }}$ as a function of spatial scale with different $\alpha$. (Right) Maximal growth rate of entanglement entropy density $\left(\frac{d \delta \overline{\mathcal{V}}}{d t_{0}}\right)$ as a function of diameter of entangled region.

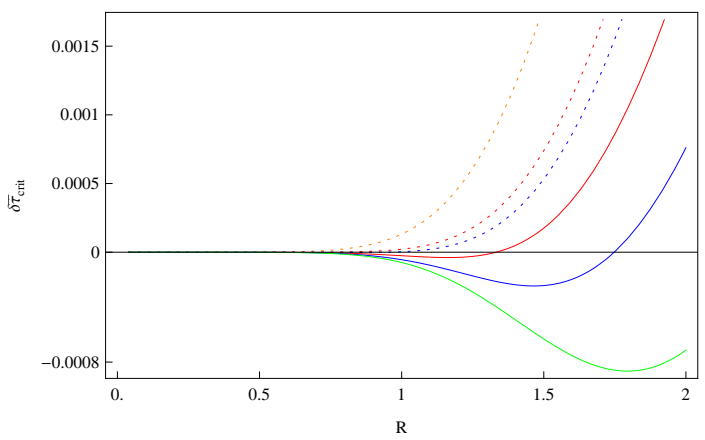

FIG. 7: The relative critical thermalization time $\delta \bar{\tau}_{c r i t}$ of EE shows certain phase transition with $\alpha=-0.05$ (orange-dashed), -0.09 (red-dashed), -0.1 (blue-dashed), -0.12 (red), -0.15 (blue), -0.19 (green). 


\section{CONCLUSION AND DISCUSSION}

In this note, using the AdS/CFT correspondence and the GB-Vaidya model we study the thermalization process of the dual boundary field theory. The two-point functions, the expectation values of circular Wilson loops and the EE with circular entangling surfaces are studied as the probes. When boundary separation $R$ (or $\ell / 2$ for two-point functions) is small, we observed that the real thermalization times of these observables have the weak dependence on the GB coupling constant $\alpha$. We also found that for two-point functions and Wilson loops, the critical thermalization time has the non-monotonic dependence on $\alpha$. For EE, the critical thermalization time always increases as $\alpha$ increases. We showed that the critical thermalization time of all three probes behave like $\tau_{\text {crit }} \sim R$ for small boundary separation $R$ but for large $R, \tau_{\text {crit }}$ is not linear with $R$. We also showed that the relationship between the critical thermalization time of EE and the boundary separation encounters certain "phase transition" at large negative $\alpha(\alpha \lesssim-0.1)$. Moreover, as in Einstein's gravity, it's found that maximal growth rate of EE density is nearly volume-independent for small volumes.

One may wonder if the larger boundary separation could lead to the new thermalization behavior. Interestingly, we find that there exist different geodesics with extremal lengths for a range of $t_{0}$. Consequently, the two-point function behaviors as a multiple-value function, see (A) in Fig. 8. We have found such kind of multiple-value functions when $\alpha$ is negative and $R$ is large enough (For instance, the similar multiple-value functions appear when $\alpha=-0.1$, $R \simeq 2.403$ and $\alpha=-0.05, R=2.4$ ). However, we have not found the similar multiplevalue functions for other two probes with the parameter spaces in which our numerical method is reliable. Moreover, we note that our numerical method is not precise enough to plot the complete thermalization process in (A) of Fig. 8. Fortunately, when $\alpha=-0.01$, $R=3.2$, we can plot the complete thermalization process, see (B) of Fig. 8, It is very interesting to observe a swallow tail, see (B) and (C) of Fig. 8 (We have also obtained the complete thermalization process with the similar swallow tail for several other parameters, for instance, the case with $\alpha=-0.03, R=2.7$ ). Comparing (A) and (C) of Fig. 8, one can suspect that the multiple-value function in (A) of Fig. 8 may be an incomplete part of a similar swallow tail. It should be pointed out that the swallow tail in the two-point function with GB correction is not same as the swallow tail observed previously in strip EE, where 
(A)

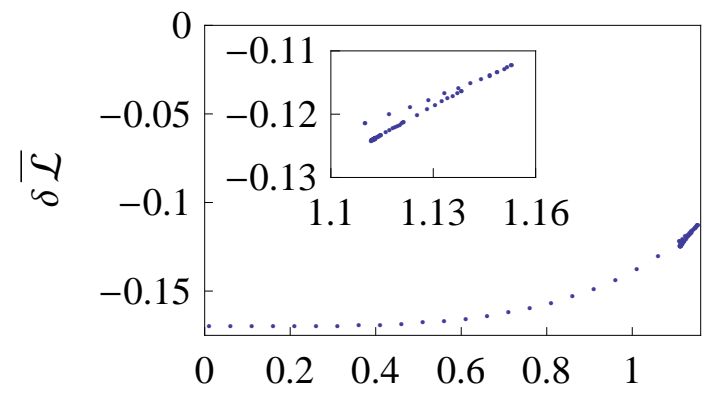

$t_{0}$

(C)

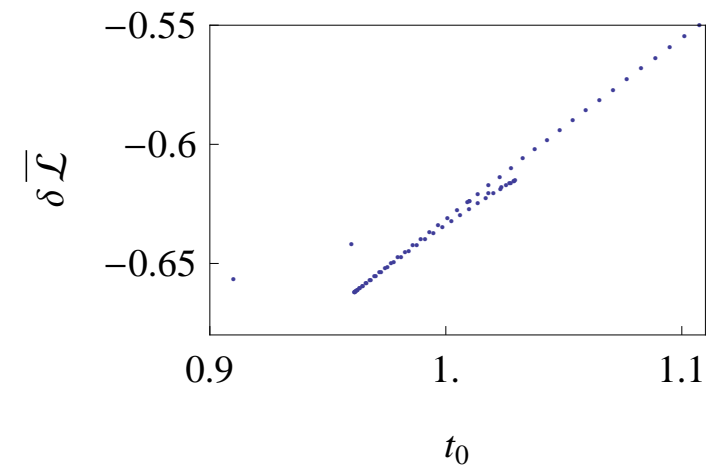

(B)

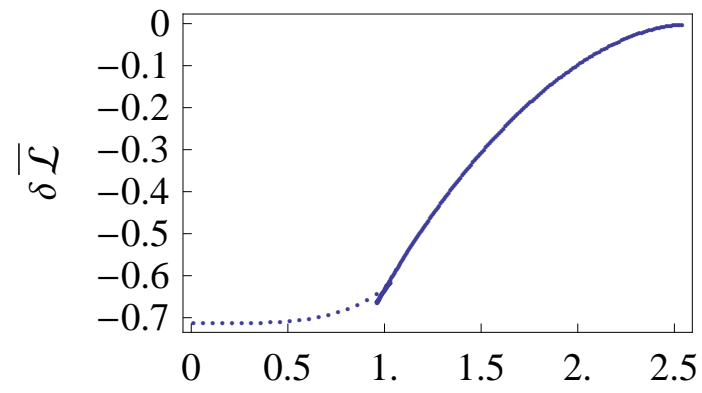

$t_{0}$

(D)

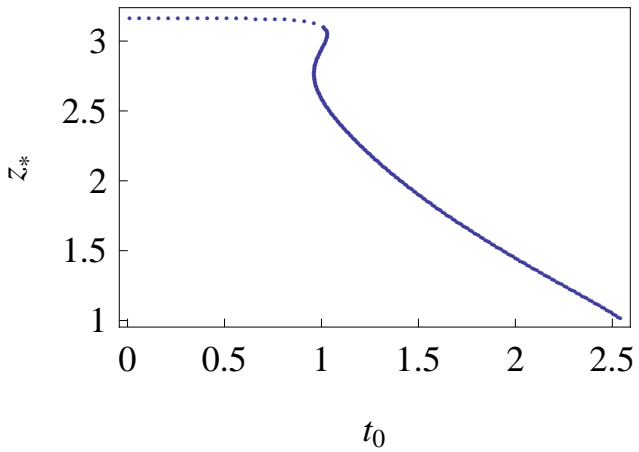

FIG. 8: (A) The two-point function with $R \simeq 2.157$ and $\alpha=-0.19$. Note that here our numerical method is not precise enough to plot the complete thermalization process. (B) The two-point function with $R=3.2$ and $\alpha=-0.01$. One can see a swallow tail. (C) A zoomed-in version of the swallow tail. (D) $z_{*}$ against $t_{0}$ for $R=3.2$ and $\alpha=-0.01$.

the swallow tail is on the top of the curve, see for instance Fig. 18 in the second reference of [20]. However, the swallow tail in Fig. 8 is on the bottom of the curve. In addition, the previous swallow tail appears near the thermalization time, but it is not the case for the swallow tail in Fig. 8. The difference between two kinds of swallow tails can also been seen by comparing the $z_{*}$ function of $t_{0}$, see (D) of Fig. 8 for the two-point function and Fig. 27 in the second reference of [22] for strip EE. The physical meaning of this new kind of swallow tail deserves to be further studied. Here we only note that the multi-valuedness of strip EE is avoided by selecting the extremal surface with the minimal area [44]. However, if we adopt the similar selection, the two-point function will be not continuous.

At last, it should be noted that in Ref. [27], the authors calculated the two-point function and rectangular Wilson loop in the field theory dual to GB gravity. So their work on the two-point function is overlapped with ours. However, they did not notice the swallow tail 

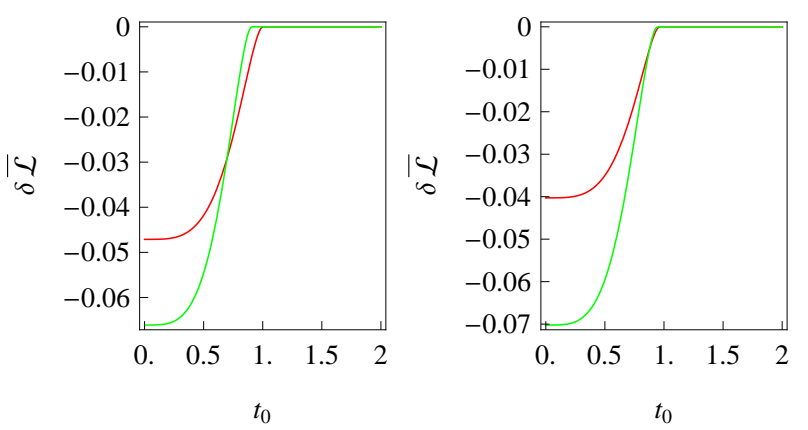

FIG. 9: Thermalization of the renormalized geodesic lengths using the metric (2.14) of Ref. [27]. The green and red lines correspond to $\alpha=0.08$ and -0.1 , respectively. (Left) The boundary separation $\ell=2$ (see Fig. 2 in [27]). (Right) $\ell=2 / L_{A d S}$. One can see that the dependence of the thermalization time on $\alpha$ is suppressed by the scale transformation.

which is present outside the parameters space they scanned. Moreover, they did not show that the thermalization times only have weak dependence on the GB coupling constant $\alpha$. Their results can be changed to ours if one makes a scale transformation on the boundary separation according to the difference between our metric and theirs, see Fig. 9.

\section{Acknowledgments}

We thank Y. P. Hu and X. H. Ge for valuable discussions. This work was supported by National Natural Science Foundation of China (No. 11275120).

[1] J.M. Maldacena, Adv. Theor. Math. Phys. 2 (1998) 231 hep-th/9711200.

[2] See following reviews: F. Gelis, J. Phys. Conf. Ser. 381 (2012) 012021 arXiv:1110.1544]; E. Iancu, arXiv:1205.0579.

[3] B. Müller, A. Schäfer, Int. J. Mod. Phys. E 20 (2011) 2235 arXiv:1110.2378.

[4] See following reviews: S. Mondal, D. Sen, K. Sengupta, arXiv:0908.2922; J. Dziarmaga, arXiv:0912.4034; A. Polkovnikov, K. Sengupta, A. Silva, M. Vengalattore, Rev. Mod. Phys. 83 (2011) 863.

[5] A. Fayyazuddin, M. Spalinski, Nucl.Phys. B 535 (1998) 219-232 hep-th/9805096; O. Aharony, A. Fayyazuddin and J. Maldacena, JHEP 9807 (1998) 013 hep-th/9806159. 
[6] O. Aharony, J. Pawelczyk, S. Theisen and S. Yankielowicz, Phys. Rev. D 60 (1999) 066001 hep-th/9901134; M. Blau, E. Gava and K.S. Narain, JHEP 9909 (1999) 018 hep-th/9904179]; Y. Kats and P. Petrov, JHEP 0901 (2009) 044 [arXiv:0712.0743].

[7] M. Brigante, H. Liu, R. C. Myers, S. Shenker and S. Yaida, Phys. Rev. Lett. 100 (2008) 191601 arXiv:0802.3318]; M. Brigante, H. Liu, R. C. Myers, S. Shenker and S. Yaida, Phys. Rev. D 77 (2008) 126006 arXiv:0712.0805].

[8] A. Buchel and R. C. Myers, JHEP 0908 (2009) 016 [arXiv:0906.2922].

[9] X. O. Camanho and J. D. Edelstein, JHEP 1004 (2010) 007 arXiv:0911.3160; A. Buchel, J. Escobedo, R. C. Myers, M. F. Paulos, A. Sinha and M. Smolkin, JHEP 1003 (2010) 111 arXiv:0911.4257]; R. G. Cai, Z. Y. Nie and Y. W. Sun, Phys. Rev. D 78 (2008) 126007 arXiv:0811.1665; X. H. Ge and S. J. Sin, JHEP 0905 (2009) 051 arXiv:0903.2527.

[10] D. M. Hofman and J. Maldacena, JHEP 0805 (2008) 012 arXiv:0803.1467]; D. M. Hofman, Nucl. Phys. B 823 (2009) 174 arXiv:0907.1625]; J. de Boer, M. Kulaxizi and A. Parnachev, JHEP 1003 (2010) 087 arXiv:0910.5347.

[11] H. Liu and S. Josephine Suh, arXiv:1305.7244.

[12] R. Gregory, S. Kanno and J. Soda, JHEP 0910 (2009) 010 arXiv:0907.3203; Q. Pan, B. Wang, E. Papantonopoulos, J. de Oliveira and A. B. Pavan, Phys. Rev. D 81 (2010) 106007 arXiv:0912.2475.

[13] A. E. Dominguez, E. Gallo, Phys. Rev. D 73 (2006) 064018 gr-qc/0512150|.

[14] J. de Boer, M. Kulaxizi, A. Parnachev, JHEP 1107 (2011) 109 [arXiv:1101.5781].

[15] L. Y. Hung, R.C. Myers, M. Smolkin, JHEP 1104 (2011) 025 arXiv:1101.5813.

[16] A. Bhattacharyya, A. Kaviraj and A. Sinha, arXiv:1305.6694.

[17] J. Abajo-Arrastia, J. Aparicio, E. Lopez, JHEP 1011 (2010) 149 [arXiv:1006.4090].

[18] J. Aparicio, E. Lopez, JHEP 1112 (2011) 082 [arXiv:1109.3571].

[19] T. Albash, C.V. Johnson, New J. Phys. 13 (2011) 045017 arXiv:1008.3027.

[20] V. Balasubramanian, A. Bernamonti, J. de Boer, N. Copland, B. Craps, E. Keski-Vakkuri, B. Müller, A. Schäfer, M. Shigemori, W. Staessens, Phys. Rev. Lett. 106 (2011) 191601 arXiv:1012.4753; V. Balasubramanian, A. Bernamonti, J. de Boer, N. Copland, B. Craps, E. Keski-Vakkuri, B. Müller, A. Schäfer, M. Shigemori, W. Staessens, Phys. Rev. D 84 (2011) 026010 arXiv:1103.2683].

[21] V. Keränen, E. Keski-Vakkuri, L. Thorlacius, Phys. Rev. D 85 (2012) 026005 
arXiv:1110.5035.

[22] D. Galante, M. Schvellinger, JHEP 1207(2012) 096 [arXiv:1205.1548]; E. Caceres, A. Kundu, JHEP 1209 (2012) 055 arXiv:1205.2354].

[23] V. E. Hubeny, M. Rangamani, E. Tonni, JHEP 1305 (2013) 136 arXiv:1302.0853.

[24] Y. Z. Li, S. F. Wu, Y. Q. Wang, G. H. Yang, JHEP 1309 (2013) 057 arXiv:1306.0210].

[25] S. A. Stricker, arXiv:1307.2736.

[26] W. H. Baron and M. Schvellinger, JHEP 1308 (2013) 035 arXiv:1305.2237].

[27] X. X. Zeng, W.B. Liu, arXiv:1305.4841.

[28] V. Balasubramanian, A. Bernamonti, J. de Boer, B. Craps, L. Franti, F. Galli, E. KeskiVakkuri, B. Müller, A. Schäfer, arXiv:1307.7086, V. Balasubramanian, A. Bernamonti, J. de Boer, B. Craps, L. Franti, F. Galli, E. Keski-Vakkuri, B. Müller, A. Schäfer, arXiv:1307.1487,

[29] S. Bhattacharyya, S. Minwalla, JHEP 0909 (2009) 034 arXiv:0904.0464.

[30] S. Lin, E. Shuryak, Phys. Rev. D 78 (2008) 125018 [arXiv:0808.0910].

[31] R. Baier, A. H. Mueller, D. Schi and D. T. Son, Phys. Lett. B 502 (2001) 51 hep-ph/0009237; A. H. Mueller, A. I. Shoshi and S. M. H. Wong, Phys. Lett. B 632 (2006) 257 hep-ph/0505164.

[32] P. Calabrese, J. Cardy, J. Stat. Mech. 0504 (2005) P04010 cond-mat/0503393.

[33] P. Calabrese, J. Cardy, J. Stat. Mech. 0706 (2007) P06008 arXiv:0704.1880.

[34] V. Latora, M. Baranger, Phys. Rev. Lett. 82 (1999) 520 chao-dyn/9806006.

[35] J. Bolte, B. Müller, A. Schäfer, Phys. Rev. D 61 (2000) 054506 hep-lat/9906037; T. Kunihiro, B. Müller, A. Ohnishi, A. Schäfer, T. T. Takahashi, A Yamamoto, Phys. Rev. D 82 (2010) 114015 arXiv:1008.1156.

[36] T. Hartman, J. Maldacena, JHEP 1305 (2013) 014 [arXiv:1303.1080].

[37] D. Steineder, S. A. Stricker and A. Vuorinen, Phys. Rev. Lett. 110 (2013) 101601 arXiv:1209.0291.

[38] D. Steineder, S. A. Stricker and A. Vuorinen, JHEP 1307 (2013) 014 [arXiv:1304.3404].

[39] R. G. Cai, Phys. Rev. D 65 (2002) 084014 hep-th/0109133.

[40] X. M. Kuang, B. Wang, J. P. Wu, JHEP 07 (2012) 125 [arXiv:1205.6674].

[41] D. V. Fursaev, JHEP 0609 (2006) 018 hep-th/0606184].

[42] H. Casini, M. Huerta, R.C. Myers, JHEP 1105 (2011) 036 arXiv:1102.0440; T. Nishioka, S. Ryu, T. Takayanagi, J. Phys. A 42 (2009) 504008 arXiv:0905.0932.

[43] M. Headrick, Phys. Rev. D 82 (2010) 126010 arXiv:1006.0047. 
[44] V. E. Hubeny, M. Rangamani and T. Takayanagi, JHEP 07 (2007) 062 arXiv:0705.0016.

[45] Note that any other curvature squared term can be reduced to GB term by field redefinitions and disregarding six or more derivatives [7].

[46] Recently the inhomogeneous holographic thermalization has been studied in [28].

[47] In Ref. 20], the maximal growth rate is used to characterize the linear growth, since the linear regime covers the time at which the growth rate is maximal.

[48] For instance, see Refs. [7, 12, 40].

[49] This can be understood as follows. At first, for smaller times $t_{0}$ the geodesics (or extremal surfaces) necessarily pass through the shell and the probes are time dependent. Second, $\tau_{\text {crit }}$ can not necessarily fix $\tau_{\text {real }}$, because even at later times there may be a geodesic which penetrates the shell and has a shorter length than the one that only extends in the region outside the shell. In short, when the shell's instantaneous position is at $z_{*}$, the geodesic may still pass through the shell. So it will need more time to reach thermal equilibrium. As an example, by studying the holographic thermalization in asymptotically Lifshitz spacetimes, the authors of Ref. 21] have found that for dynamical exponent $\tilde{z}=1, \tau_{\text {real }}$ agrees with $\tau_{\text {crit }}$ in the parameter space they scanned. But for other value of $\tilde{z}, \tau_{\text {real }}$ is indeed larger than $\tau_{\text {crit }}$ for sufficiently large $\ell$.

[50] It is not a new idea to take the boundary separation as an order parameter, see [43] for instance. 